\title{
Towards Human Level Web Intelligence: A Brain Informatics Perspective
}

\author{
Ning Zhong \\ Department of Life Science and Informatics \\ Maebashi Institute of Technology, Japan \& \\ The International WIC Institute \\ Beijing University of Technology, China \\ zhong@maebashi-it.ac.jp
}

In this talk, we outline a vision of Web Intelligence (WI) research from the viewpoint of Brain Informatics (BI), a new interdisciplinary field that studies the mechanisms of human information processing from both the macro and micro viewpoints by combining experimental cognitive neuroscience with advanced information technology. BI can be regarded as brain sciences in WI centric IT age and emphasizes on a systematic approach for investigating human information processing mechanism. Advances in instruments like fMRI and information technologies offer more opportunities for research in both Web intelligence and brain sciences. Further understanding of human intelligence through brain sciences fosters innovative Web intelligence research and development. Web intelligence portal techniques provide a powerful new platform for brain sciences. The synergy between WI and BI advances our ways of analyzing and understanding of data, knowledge, intelligence and wisdom, as well as their relationship, organization and creation process. Web intelligence is becoming a central field that changes information technologies, in general, and artificial intelligence, in particular, towards human-level Web intelligence. 